\title{
Generating steep, shear-free gradients of small molecules for cell culture
}

\author{
Taesung Kim • Mikhail Pinelis • Michel M. Maharbiz
}

Published online: 8 August 2008

(C) Springer Science + Business Media, LLC 2008

\begin{abstract}
We present the fabrication, characterization and cell culture results of a microfluidic device for generating steep gradient interfaces of small molecules $(<1 \mathrm{kDa})$ across cell culture with no convective shear stresses applied to the cells. We use a novel streamline of two fluids to generate stable and uniform gradient interfaces/boundaries by confronting one fluid with the other. We separate a gradient generation channel and a cell culture channel by a polyester membrane so that viscous shear stress by the bottom channel flow does not convectively disturb the chemical environment of cultured cells seeded on the membrane in the top channel. Using two-component dyes to characterize the steepness of the diffusional interface, we demonstrate $50 \mu \mathrm{m}$ wide steps for about 400 Da molecules. Using BCECF, a 689 Da pH-sensitive diffusible dye which is actively taken up by living cells, we demonstrate gradient boundaries narrower than five cell diameters in HeLa culture. We also demonstrate steep gradients of $\mathrm{pH}$ across cells in the same device. This work should be of interest to researchers attempting to generate gradients of small, rapidly diffusing molecules for studies in cellular differentiation and signaling.
\end{abstract}

Keywords Small molecules · Gradient · Membrane ·

Microfluidics $\cdot$ HeLa cell culture

T. $\operatorname{Kim}(\bowtie) \cdot$ M. M. Maharbiz

Electrical Engineering and Computer Science,

University of California at Berkeley,

Berkeley, CA 94720-1770, USA

e-mail: taesungk@eecs.berkeley.edu

M. Pinelis

Electrical Engineering and Computer Science,

University of Michigan,

1301 Beal Ave.,

Ann Arbor, MI 48109, USA

\section{Introduction}

In developmental biology, diffusible signal molecules play a key role in inducing and timing proliferation, differentiation, and migration of cells (Ashe and Briscoe 2006; Gurdon and Bourillot 2001; Lander 2007). Since controlling and sensing the gradients of signaling molecules provides a basis for understanding many patterning/developmental processes, there has been much interest in microfluidic devices for generating gradients of such molecules (Campbell and Groisman 2007; Chung et al. 2005; Dertinger et al. 2001; Irimia et al. 2006; Jeon et al. 2002; Keenan and Folch 2008; Takayama et al. 2001). These devices have attempted to recreate in vivo cellular chemical environments in vitro by exploiting microscale mass transfer phenomena, including manipulation of laminar flows (Chung et al. 2005; Irimia et al. 2006; Jeon et al. 2000; Saadi et al. 2006; Wang et al. 2006), generation of mass-transfer limitations in microchannels (Abhyankar et al. 2006; Keenan et al. 2006; Yu et al. 2005), and microscale dosing (Bansal et al. 2007; Chung et al. 2006). These devices have the potential to generate microenvironments that more closely approximate the in vivo milieu (El-Ali et al. 2006; Khademhosseini et al. 2006) than traditional, two chamber techniques such as Boyden (Boyden 1962), Zigmond (Zigmond 1977), and Dunn (Zicha et al. 1991) chambers. However, existing microfluidic gradient generation devices have known drawbacks; those that depend on convective flow and diffusive mixing within channels populated by cells tend to remove secreted chemicals even as they generate gradients and expose the cells to shear forces (Chung et al. 2005; Irimia et al. 2006; Jeon et al. 2000; Saadi et al. 2006; Wang et al. 2006). Microfluidic methods that do not employ flow eliminate or reduce shear, but tend to generate shallow gradients and lack the ability to produce complex gradient 
profiles (Abhyankar et al. 2006; Keenan et al. 2006; Yu et al. 2005). Recently, a class of devices has emerged which cultures cells across a diffusible membrane so that the interference with hydrodynamic flow is minimized (Abhyankar et al. 2006; Chueh et al. 2007). However, it seems that an attempt that combines the advantage of microscale convective flow to generate gradients with that of a membrane to eliminate/reduce shear stresses on cells has not been made to date.

The principal goal of the present work was to design and characterize a shear-free gradient generator for very small, rapidly diffusing molecules. Attempts at eliciting sharp graded cell responses to diffusible cues have been limited by the fact that many signals (including signals secreted by the cells themselves, such as Lewis acids, bases and small peptides) diffuse rapidly in cell culture (Gurdon and Bourillot 2001; Lander et al. 2002; Yu et al. 2005). One of the most extreme cases, the hydroxyl ion, has a diffusion constant greater than: $\sim 5.28 \times 10^{-9} \mathrm{~m}^{2} / \mathrm{s}$ in water (Cussler 1997).

Here we present a new method for small molecules that builds on the advantages of convection flow-based devices and those of diffusion-based devices. Our device maps chemical gradients generated in a novel gradient generation chamber across a diffusible membrane onto an overlying cell culture chamber (Fig. 1). The membrane prevents convective flow while allowing for diffusion, so that viscous shear stress from the bottom channel flow does not interfere with cells attached on the top of the membrane. Because morphogen gradients can arise in vivo across very short distances $(\sim$ a few tens micrometer; Gurdon and Bourillot 2001; Kicheva et al. 2007; Raftery and Sutherland 2003; Shimmi and O'Connor 2003) but

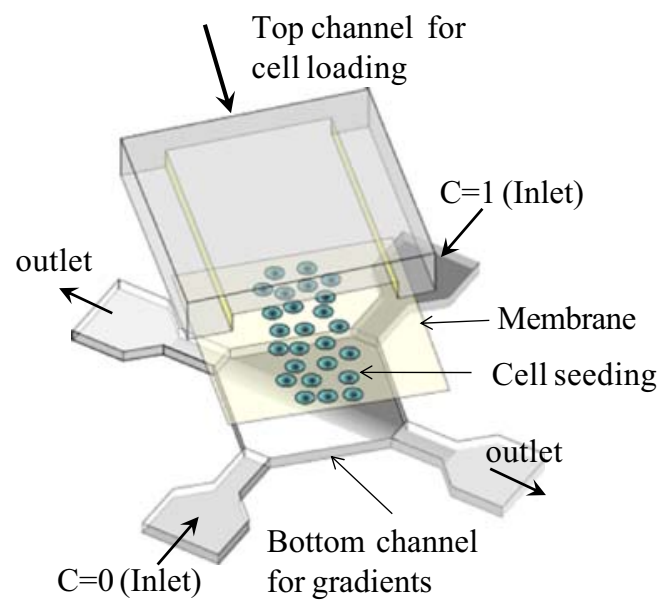

Fig. 1 Schematic view of the device. The top channel contains cells in culture medium and is separated from the bottom channel by a $10 \mu \mathrm{m}$ thick polyester membrane. The bottom channel produces sharp gradients of small molecules by directly confronting flows from the two inlets. The generated conditions map across the membrane into the cells in culture depend on mechanisms that span much larger collections of cells (e.g., the size of an embryo), we sought to generate steeper gradients over a larger span of cells than conventional Y-shaped channels. In our method, two flows with different chemical concentrations of the molecule of interest confront each other; the resulting convective flow creates a very sharp boundary across the cell culture. Due to the low Reynolds number of the fluid, the fluid confrontation maintains a uniform mixing zone along the fluid boundary with a steep, stable and uniform gradient across this boundary. Because typical Y-shaped microfluidic channels have progressively worsening interfaces as diffusion and mixing progress downstream along the channel, the presented method provides a unique advantage for applications with fast diffusing components (Nie et al. 2007; Takayama et al. 2001; Walker et al. 2005).

In this work, we first present characterization of gradients generated in the bottom channel using a fluorescent dye and compare the result with other published devices. Second, we quantify the extent of mixing of our device at the interface using free $\mathrm{Ca}^{2+}$ and a $\mathrm{Ca}^{2+}$-activated dye (Fluo-5N) in order to compare the result with that obtained in a typical Y-shaped channel under the same flow rates. Third, we demonstrate how generated concentration differences are mapped onto membrane-cultured HeLa cells using BCECF, a $689 \mathrm{Da}$ fluorescent molecule which is actively taken up by living cells. Since one of the most extreme cases, the hydroxyl ion, has a diffusion constant of $D_{\mathrm{OH}^{-}}=\sim 5.28 \times 10^{-9} \mathrm{~m}^{2} / \mathrm{s}$ in water (Cussler 1997), we generate two regions of distinct $\mathrm{pH}$ and then demonstrate how this graded condition maps to cells cultured on the membrane. Finally, we demonstrate how we can actively move the interface boundary within the device and produce asymmetric interface regions by manipulating flow rates at inlets and outlets.

\section{Experimental}

\subsection{Reagents}

Chemical gradients were characterized with rhodamine-B (Invitrogen, $400 \mathrm{Da}$ ) and Fluo-5N pentapotassium salt (Invitrogen, $996 \mathrm{Da}$ ). A $100 \mu \mathrm{M} \mathrm{CaCl}_{2}$ (Invitrogen) solution was made with DI water. BCECF AM (Invitrogen, $689 \mathrm{Da}$ ) was used to stain cells and to indicate intracellular $\mathrm{pH}$ based on the manufacturer's instructions. Different $\mathrm{pH}$ solutions were made from distilled water $(\mathrm{pH}=6.6)$, PBS buffer (Phosphate-Buffered Salines, $\mathrm{pH}=7.6$, Invitrogen), and HBSS buffer (Hanks' balanced salt solutions, $\mathrm{pH}=8.54$, Invitrogen). Dulbecco's modified Eagle's medium (DMEM, Invitrogen) containing $15 \% v / v$ fetal bovine serum (FBS, Gibco) and $4 \mathrm{mM}$ L-glutamine (Invitrogen) was used for cell culture. 


\subsection{Device fabrication}

The device was fabricated from two layers of (poly) dimethylsiloxane (PDMS) (Sylgard 184, Dow Corning, Midland, MI, USA) and a polyester membrane $(0.4 \mu \mathrm{m}$ in pore diameter, Corning Inc., New York). The membrane attachment process is a refinement of a method by Chueh et al. (2007) and Ismagilov et al. (2001). Briefly, the bottom $(600 \times 600 \times 50 \mu \mathrm{m}, W \times L \times D)$ and the top channel $(500 \mu \mathrm{m}$ in width and $80-100 \mu \mathrm{m}$ in depth) layer were made by casting PDMS on a SU-8 50 (MicroChem, Newton, MA, USA) mold, respectively (Duffy et al. 1998). Both layers were then stamped with PDMS pre-polymer coated coverslips (3:2 mixing ratios of Toluene to PDMS by weight) to selectively transfer the PDMS pre-polymer to the junction areas except the channels. Subsequently, a piece of the membrane $(3 \times 3 \mathrm{~mm})$ was laid over the top channel layer, which was aligned with the bottom channel layer using a bright field microscope (Nikon, SMZ 200). The aligned top and bottom layers were heated in a $120{ }^{\circ} \mathrm{C}$ oven for $30 \mathrm{~min}$ to cure the PDMS pre-polymer and then pressure was applied using regular office clips for another 30 min under the same temperature. This two step curing process produced a tight seal with no leakage and removed air bubbles trapped between the top and bottom layer. Prior to testing, the input and output ports were connected to a syringe pump (KD Scientific, Fisher Scientific International Inc.) with Tygon ${ }^{\circledR}$ tubing (Medical/Surgical Tubing, United States Plastic Corp., Lima, OH, USA).

\subsection{Experimental setup for gradient generation for $\mathrm{HeLa}$ cells}

An inverted fluorescence microscope (Nikon, TE2000) equipped with a CCD camera (Hamamatsu, Orca-ER, Japan) was used to record fluorescence signals coming from dyes; rhodamine-B dye was excited at $530 \mathrm{~nm}$ and Fluo-5N was excited at $430 \mathrm{~nm}$, respectively. For Fluo-5N, images were taken at $27 \mathrm{~ms}$ exposure. For cell culture experiments, the top channel and the membrane were coated with fibronectin $(40 \mu \mathrm{g} / \mathrm{ml})$ for $10 \mathrm{~min}$, dried out overnight and then sterilized under UV light for $30 \mathrm{~min}$. Both channels were then flushed with $\sim 1 \mathrm{ml}$ of DMEM media. HeLa cells (carcinoma cancer cells) were then loaded into the top channel. Cells were incubated at $37^{\circ} \mathrm{C}$ in a $5 \% \mathrm{CO}_{2}$ atmosphere humidified incubator for $10 \mathrm{~h}$. Cells clearly attached and spread on the membrane. During experiments, devices were moved to the inverted microscopy stage and the relevant reagents were flown through the channels while imaging. All image processing was conducted using Image J. BCECF pH levels were calculated from ratios of fluorescent intensities of BCECF-stained cells taken at two different excitation wavelengths (430 and
$490 \mathrm{~nm}$ ) and calibrated to pHi levels using published calibration curves (Weiner and Hamm 1989). Experimental results were supported by performing FEMLAB simulations (Version 3.3a, Comsol Inc., Massachusetts, USA).

\section{Results and discussion}

\subsection{Sharp gradients can be generated over large areas}

Two fluid flows meet each other in the center of the device, creating a boundary parallel with the diagonal of the square bottom chamber. In order to characterize gradient profiles for various flow velocities, we flowed diffusible fluorescent dye (rhodamine-B, $D_{\mathrm{rh}}=3.6 \times 10^{-10} \mathrm{~m}^{2} / \mathrm{s}$; Rani et al. 2005 ) in DI water into one inlet and DI water into the other (Fig. 2). Since slow flow rates allow longer diffusion time across the interface, faster flows produce sharper gradients [Fig. 2(c) and (d)].

Figure 2(b) quantifies fluorescence intensities along the $x$-axis under different flow rates ranging from 0.01 to $1 \mathrm{ml} / \mathrm{h}$. In order to quantitatively compare the steepness of our interfaces with others, we defined a value $x_{90-10}$ as the distance the gradient falls between $90 \%$ and $10 \%$ of the maximum intensity. This metric is common in domains with sigmoidal or exponential transitions (e.g., transistor switching times). For the device in Fig. 2 , the $x_{90-10}$ distances were 54,36 and $23 \mu \mathrm{m}$ for $0.01,0.1$ and $1 \mathrm{ml} / \mathrm{h}$ flow rates, respectively. These distances are approximately 5-20× smaller than comparable $x_{90-10}$ distances calculated for existing technology employing a similar range of molecular weights and flow rates (Table 1). Note that in a Y-shaped channel, the steepness of the chemical gradient decreases along the axis of fluid flow away from the inlets; for this reason, we carried out control experiments against Y-shaped channels (see below).

\subsection{Sharp gradients can be generated for small molecules}

We employed Fluo-5N to visualize the diffusion and mixing of very small molecules. Fluo- $5 \mathrm{~N}$ is a $\mathrm{Ca}^{2+}$ indicator, fluorescing only when it reacts with free $\mathrm{Ca}^{2+}$. Again, faster flow rates generated a steeper gradient and, thus, a narrower interface region as shown in Fig. 3(a) and (b). Given that $\mathrm{Ca}^{2+}\left(40 \mathrm{Da}, D_{\mathrm{Ca} 2+}=\sim 2.7 \times 10^{-9} \mathrm{~m}^{2} / \mathrm{s}\right.$; Horvath 1985) diffuses much faster than the Fluo-5N molecule $\left(996 \mathrm{Da}, D_{\text {fluo-5N }}=\sim 5 \times 10^{-10} \mathrm{~m}^{2} / \mathrm{s}\right.$ which was estimated from similar molecules; Rani et al. 2005), we obtained an asymmetric interface region [Fig. 3(c)]. The resulting thickness $(\delta)$ of the interface region, defined as the distance from maximum intensity to the point at which signal dropped below the noise background for our exposure, was measured to be 40 and $80 \mu \mathrm{m}$ at $1 \mathrm{ml} / \mathrm{h}$ and $0.01 \mathrm{ml} / \mathrm{h}$ flow 
Fig. 2 (a) Micrograph of the device showing each inlet and outlet port of the bottom channel. Since the microfluidic channel network is designed to confront one fluid from $Q_{2}$ with another fluid from $Q_{3}, Q_{1}$ and $Q_{4}$ have the same flow rates. (b) The normalized fluorescence intensities of three different flow rates along the $x$-axis quantify gradients of fluorescent dye (Rhodamine-B, $400 \mathrm{Da}$ ). The distance between $90 \%$ and $10 \%$ of maximal intensity, $x_{90-10}$, is discussed in the text. Subpanels (c) and (d) show fluorescent images at different flow rates. The streamlines of two fluids are symmetrical along the $y$-axis so that chemical gradients are generated along the $x$-axis

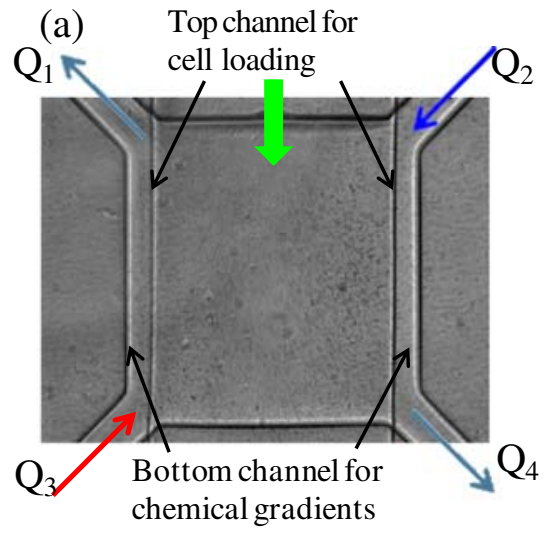

(b)
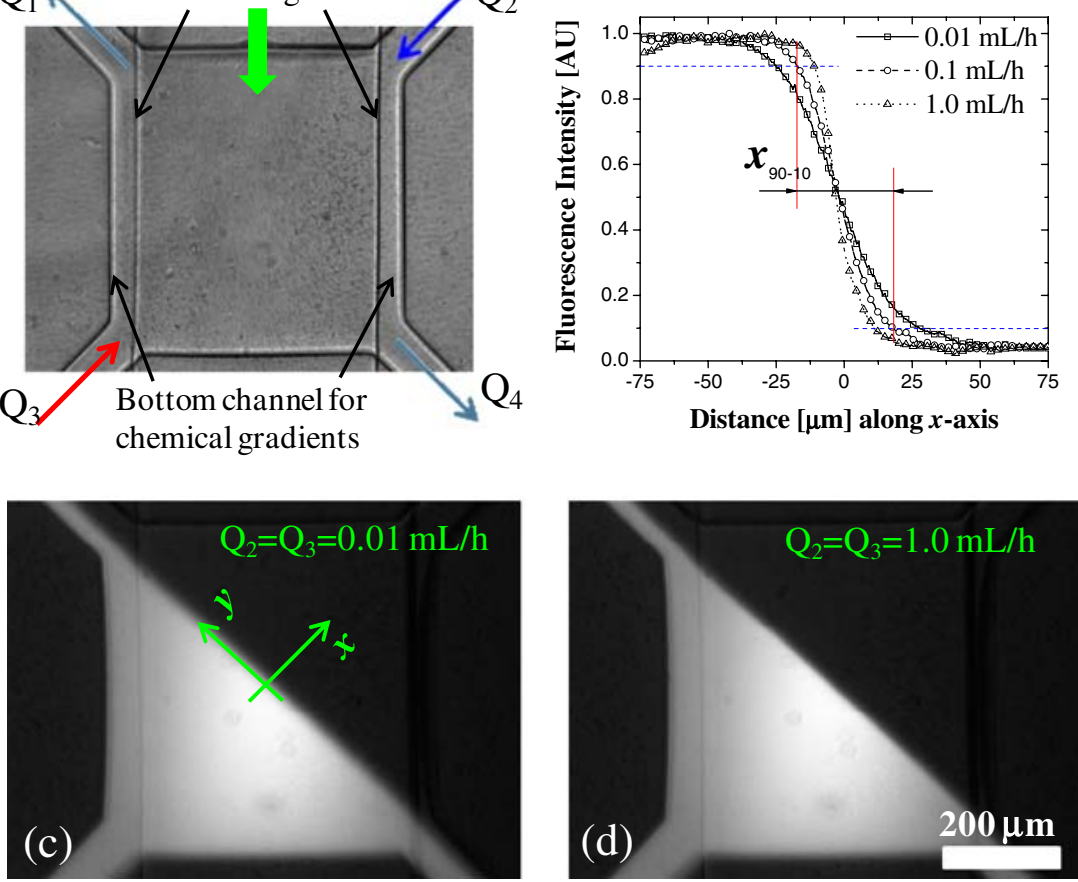

rate, respectively. The diffusion time, $t$, for $\mathrm{Ca}^{2+}$ can be approximated to $0.2-0.6 \mathrm{~s}$ using $\delta$ the diffusion distance $\left(t=\delta^{2} /\left(4 D_{\mathrm{Ca} 2+}\right)\right)$. Since the diffusion time is much larger than the reaction time $(\sim 1 \mathrm{~ms})$ of Fluo-4 with $\mathrm{Ca}^{2+}$ (Bringer et al. 2004), which has very similar properties to Fluo- $5 \mathrm{~N}$, the assumption that reaction is much faster than diffusion seems reasonable. We also varied the flow rates to each channel $\left(Q_{2}=0.1 \mathrm{ml} / \mathrm{h}\right.$ and $Q_{3}=1.0 \mathrm{ml} / \mathrm{h}$ and vice versa), showing that it is possible to actively manipulate the center position of the diffusion/mixing boundary, as shown in Fig. 3(d) and (e). In this way the center of the interface region can be swept across the cell culture area during the course of an experiment. FEM simulations support the empirical results of Fig. 3(b) and (e). Figure 3(f) shows simulated profiles for a large range of flow ratios between the two inlet channels $\left(Q_{2} / Q_{3}=1,3\right.$, and 10), showing the possible profiles for our geometry while the ratio of the two outlet channels remains constant $\left(Q_{1} / Q_{4}=1, Q_{1}+Q_{4}=Q_{2}+Q_{3}=2 \mathrm{ml} / \mathrm{h}\right)$.

\subsection{Comparison to Y-shaped channels}

In addition to generating steep gradient of small molecules, the device enables a steep, uniform interface for a longer distance along the diagonal than would be generated for a comparable Y-shaped device. To quantify this, we performed a control experiment between a conventional Y-shaped channel of similar dimensions $(\sim 50 \mu \mathrm{m}$ in depth and $500 \mu \mathrm{m}$ in width) and our device. Additionally, the actual $\mathrm{Ca}^{2+}$ gradient in our device might be wider than quantified in Fig. 2 unless all $\mathrm{Ca}^{2+}$ reacted out with the Fluo5N indicator; the control experiment

Table 1 Molecular weights, $x_{90-10}$ distances, and flow rates used in various studies

\begin{tabular}{lllll}
\hline Molecule & $\mathrm{MW}^{\mathrm{a}}(\mathrm{Da})$ & $x_{90-10}(\mu \mathrm{m})$ & Flow rates $(\mathrm{ml} / \mathrm{h})$ & Ref. \\
\hline Rhodamine-B & 400 & $54,36,23$ & $0.01,0.1,1.0$ & This work \\
FITC dextran & $10 \mathrm{k}$ & 800 & 0.0001 & Chung et al. (2005) \\
Fluorescein & 390 & 400 & 0.003 & Irimia et al. (2006) \\
Interleukin-8 & $8 \mathrm{k}$ & 150 & 0.09 & Jeon et al. (2002) \\
Fluorescein & 390 & 700 & 0.108 & Campbell and Groisman (2007) \\
Fluorescein & 390 & 640 & 0.122 & Zhu et al. (2004) \\
Fluorescein & 390 & 100 & $0.50^{\mathrm{b}}$ & Dertinger et al. (2001) \\
Green/Blue dextran & $3-70 \mathrm{k}$ & & Ko flow & Keenan et al. (2006)
\end{tabular}

${ }^{\mathrm{a}} \mathrm{MW}$ indicates the molecular weight used in characterizing gradients.

${ }^{\mathrm{b}}$ The depth of the channel was assumed to be about $50 \mu \mathrm{m}$. 

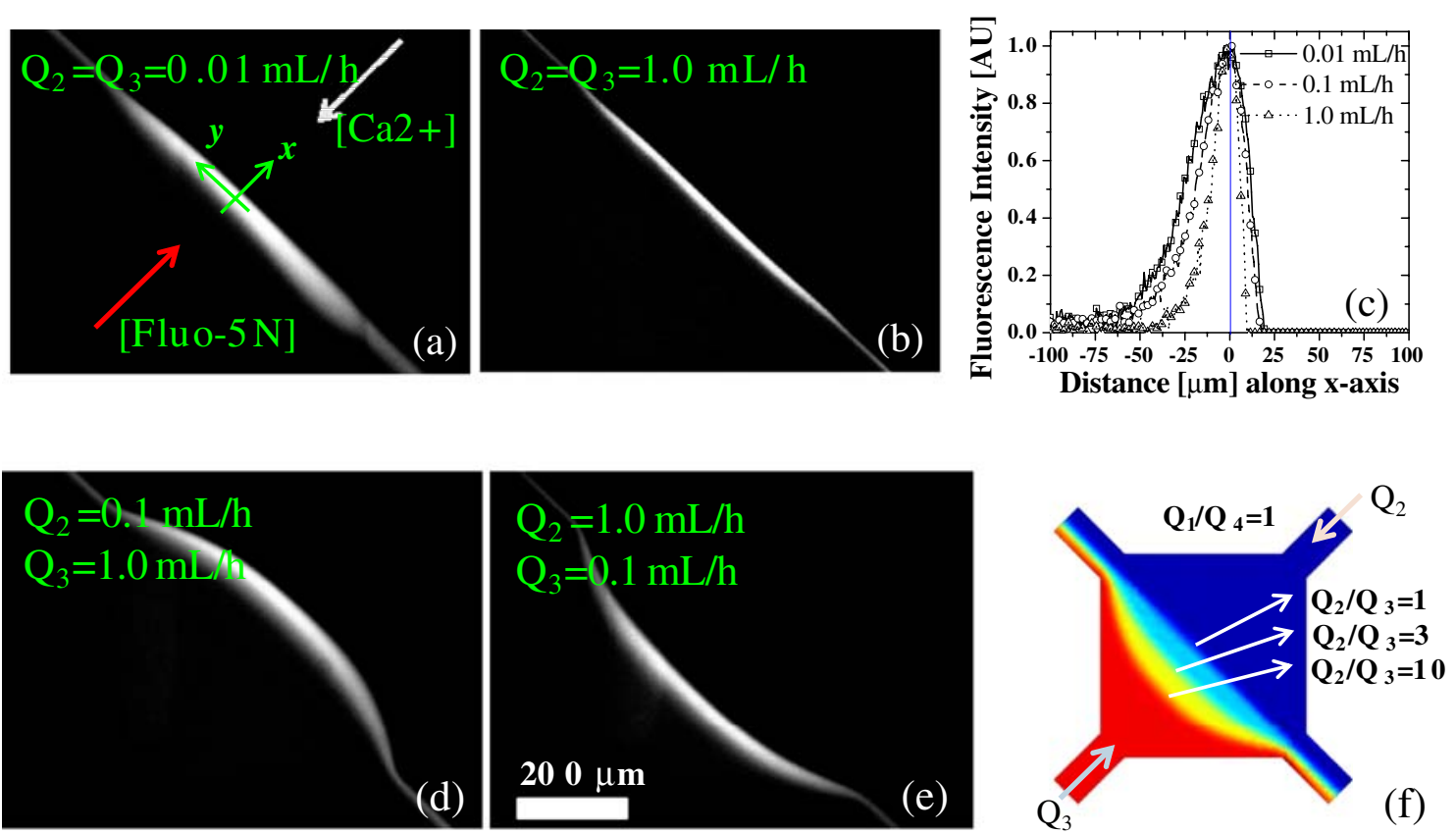

Fig. 3 Characterization of $\mathrm{Ca}^{2+}\left(D_{\mathrm{Ca}^{2+}}=\sim 3 \times 10^{-9} \mathrm{~m}^{2} / \mathrm{s}, 40 \mathrm{Da}\right)$ gradients using Fluo-5N (996 Da). Symmetric gradients (a) and (b) and the normalized fluorescence intensities of three different flow rates along the $x$-axis quantify gradients of $\mathrm{Ca}^{2+}(\mathbf{c})$. Asymmetric gradients along the boundary of two fluids are achieved by controlling flow rates (d) and

(e). Subpanel (f) shows simulated profiles for a large range of flow ratios; $Q_{2}=Q_{3}=0.1 \mathrm{ml} / \mathrm{h}\left(Q_{2} / Q_{3}=1\right), Q_{2}=0.3 \mathrm{ml} / \mathrm{h}$ and $Q_{3}=0.1 \mathrm{ml} / \mathrm{h}\left(Q_{2} /\right.$ $\left.Q_{3}=3\right)$, and $Q_{2}=1.0 \mathrm{ml} / \mathrm{h}$ and $Q_{3}=0.1 \mathrm{ml} / \mathrm{h}\left(Q_{2} / Q_{3}=10\right)$ for all cases $Q_{2}+Q_{3}=Q_{1}+Q_{4}$. FEM simulations show good match with experimental results

provided a means to quantify the same reaction in two different devices.

As expected, the extent of diffusion and mixing along the Y-channel increases downstream due to longer diffusion

time $\left(\delta \sim(D t)^{1 / 2}\right)$, generating an ever-widening mixed region [Fig. 4(a) and (c)]. In our device, the two fluids have velocities initially normal to the interface (at the center) and the streamline gradually becomes parallel with the diagonal

Fig. 4 (a) For a typical Yshaped microfluidic channel $(50 \mu \mathrm{m}$ in depth and $500 \mu \mathrm{m}$ in width), the interface area gets wider along the streamline of the two fluids $(Q=0.05 \mathrm{ml} / \mathrm{h}$ for each) because $\mathrm{Ca}^{2+}$ ions and Fluo-5N molecules diffuse into each other. The scale bar is $500 \mu \mathrm{m}$. (b) In our device, a sharper, more uniform diffusion area is produced along the boundary of two fluids $\left(Q_{2}=\right.$ $Q_{3}=0.05 \mathrm{ml} / \mathrm{h}$ ) by the convective flows initially normal to the interface. The scale bar is $200 \mu \mathrm{m}$. Subpanels (c) and (d) quantifies fluorescence intensities of (a) and (b), respectively (a)

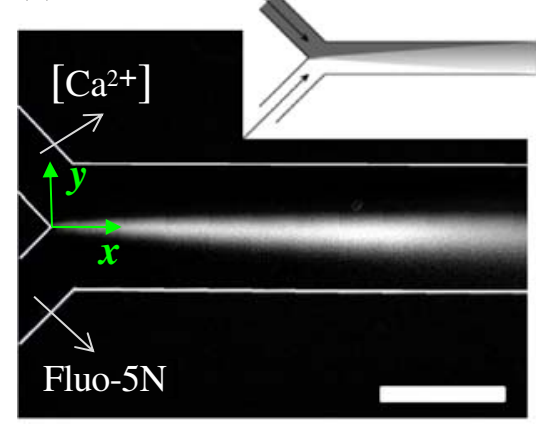

(c)

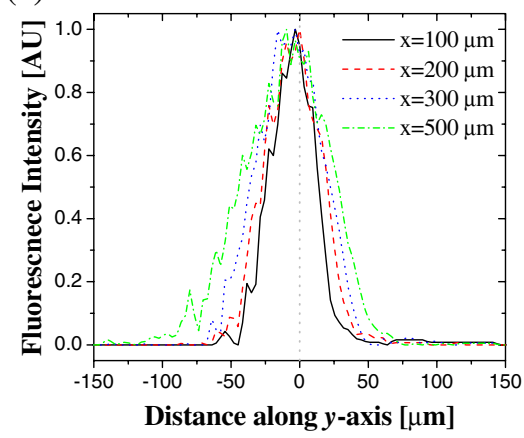

(b)

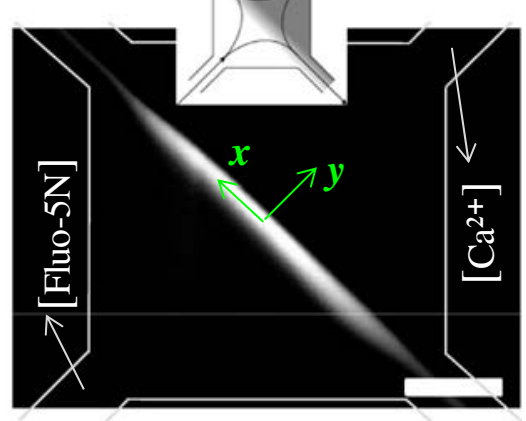

(d)

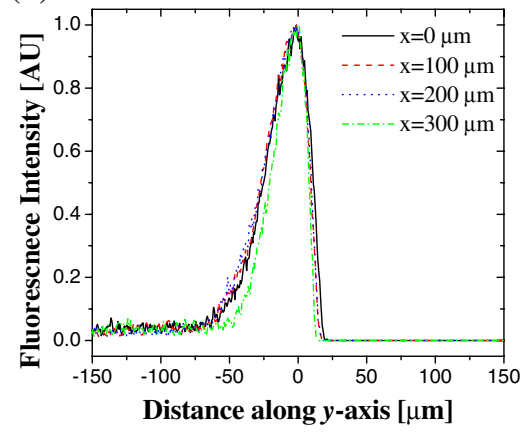


line as one moves to the exit ports. The normal, convective components generate a diffusion/mixing area that is quite uniform [Fig. 4(b) and (d)]; about $600 \mu \mathrm{m}(x=-300 \mu \mathrm{m}$ to $x=$ $300 \mu \mathrm{m})$ out of $700 \mu \mathrm{m}\left(=500 \times 2^{1 / 2} \mu \mathrm{m}\right.$, the diagonal distance of the device) can experience the same stable interface. As mentioned above, we obtained the asymmetric distribution of the fluorescence intensity resulting from the different diffusion constants of Fluo-5N molecules and $\mathrm{Ca}^{2+}$ ions.

Note that by scaling both the inlet channels and the width of the chamber, it should be possible to scale up this design to provide larger interface areas. This is not possible with Y-channel mixers.

\subsection{A 689 Da molecule gradient can be mapped across} the membrane onto HeLa cells in culture

In order to characterize how the lower chamber gradients mapped across the membrane and onto the upper, cell culture chamber, we used adherent cultures of HeLa cells and BCECF, an indicator the fluoresces only when taken up by cells (Elsabban and Pauli 1991; Weiner and Hamm 1989). For the experiments, cells in medium suspension were loaded into the top channel, allowed to settle, and then provided nutrient media using continuous flow across the bottom channel overnight $\left(Q_{2}=Q_{3}=0.1 \mathrm{ml} / \mathrm{h}\right)$. As seen in Fig. 5(a), HeLa cells grew and attached on the cell membrane overnight. We then ran a solution of BCECF AM diluted with the DMEM media through one port $\left(Q_{3}=\right.$ $0.1 \mathrm{ml} / \mathrm{h}$ ) and a solution of media through the other port $\left(Q_{2}=0.1 \mathrm{ml} / \mathrm{h}\right.$, creating different regions as in Figs. 2 and 3$)$. BCECF AM is non-fluorescent, but is uptaken by viable cells entirely within $30 \mathrm{~min}$ and converted to BCECF, which fluoresces; this dye is a common indicator of intracellular $\mathrm{pH}$ and used in uptake assays (Elsabban and Pauli 1991; Weiner and Hamm 1989). After 60 min, a BCECF pattern matching the underlying flow gradient was clearly visible in the cells [Fig. 5(b) and (c)]. Figure 5(d) quantifies the gradient of BCECF; at $t=0$, the BCECF AM gradient was generated across the bottom channel and no fluorescence was seen in the cells. The fluorescence intensity between $x=-300 \mu \mathrm{m}$ and $x=0 \mu \mathrm{m}$ gradually increased with time, indicating that cells took up BCECF $\mathrm{AM}$ molecules. The fluorescence intensity in cells lying between $x=0 \mu \mathrm{m}$ and $x=300 \mu \mathrm{m}$ did not increase above the background. The fluorescence is discretized inside the cells; the observed steepness of the interface profile in Fig. 5(d) corresponds to one cell diameter. This experimental result confirms that steep gradients generated by the bottom channel are mapped to cells attached on the membrane. Given that the membrane has $400 \mathrm{~nm}$ pores spanning a $10 \mu \mathrm{m}$ thickness (an aspect ratio of 25:1), the hydraulic resistance of the pore is so high that the convective flow generated through the membrane by flow tangential to the membrane surface is negligible (Chellam et al. 1992).

We chose a cell-dependent uptake assay for several reasons. First, it was difficult to distinguish the fluorescent signals from fluorophore concentrations in the top and bottom chambers. Inverted fluorescent microscopy did not have a narrow enough depth of focus to resolve the top channel independent of the bottom one. The device was too thick for confocal fluorescent microscopy. Second, an uptake assay is a more onerous test of gradient mapping as the total fluorescent signal is a time integral of the number of molecules that have diffused across and been
Fig. 5 (a) White light micrograph of HeLa cells densely seeded on the polyester membrane. As in Fig. 2, two regions were created in the underlying channel: one flow contained BCECF AM in DMEM and one contained DMEM. As cells took up BCECF AM across the membrane, they produced BCECF (which fluoresces). (b) and (c) Fluorescent images after 60 and 120 min show the two different conditions generated in the cells. (d) Plotted fluorescent concentrations across the boundary quantify the sharpness of the gradient
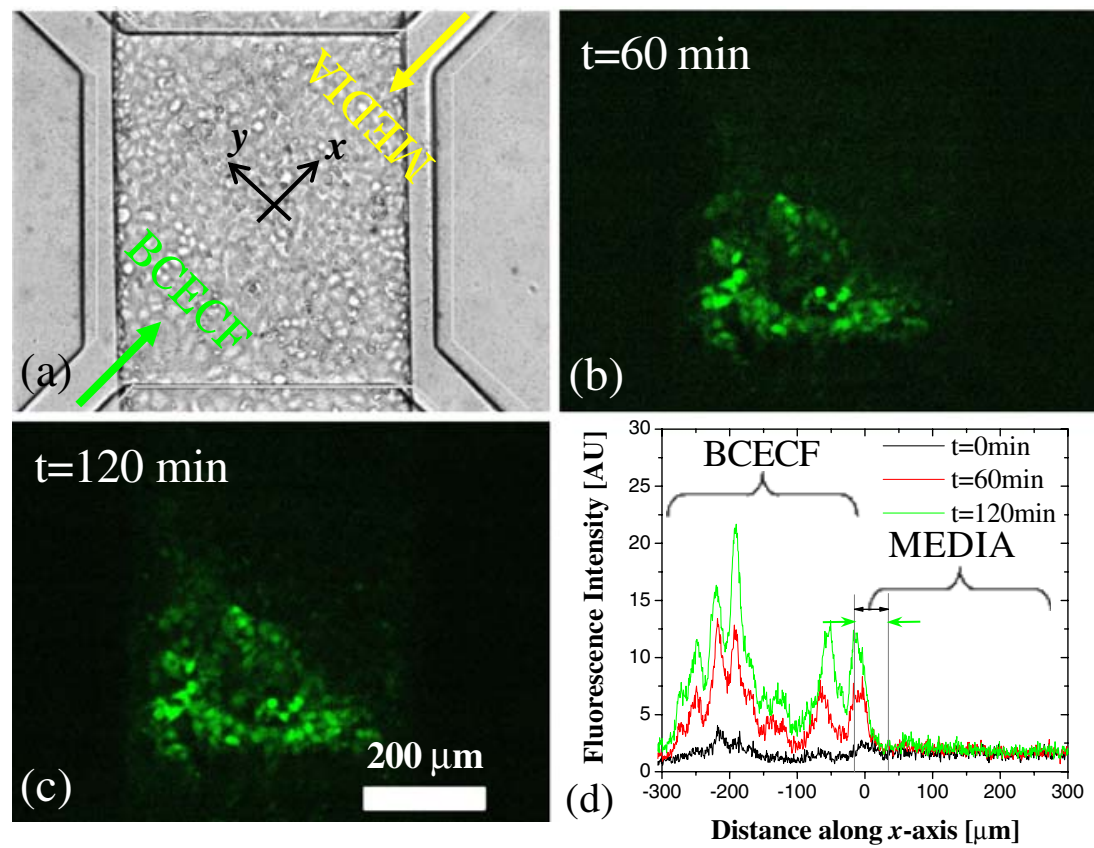
Fig. $6 \mathrm{HeLa}$ cells were loaded into the top channel, allowed to adhere to the polyester membrane, then allowed to take up BCECF AM for $30 \mathrm{~min}$. Free BCECF AM was then rinsed away from the cells with PBS buffer solution $(\mathrm{pH}=7.6)$. Once taken up by cells, the ratio of BCECF fluorescence at $490 \mathrm{~nm} /$ $430 \mathrm{~nm}$ is linearly proportional to pHi. (a) Ratiometric image at $t=0 \mathrm{~min},(\mathbf{b})$ ratiometric image at $t=120 \mathrm{~min}$, showing two zones of pHi. (c) Profiles of ratiometric fluorescence intensities along the $x$-axis as a function of time
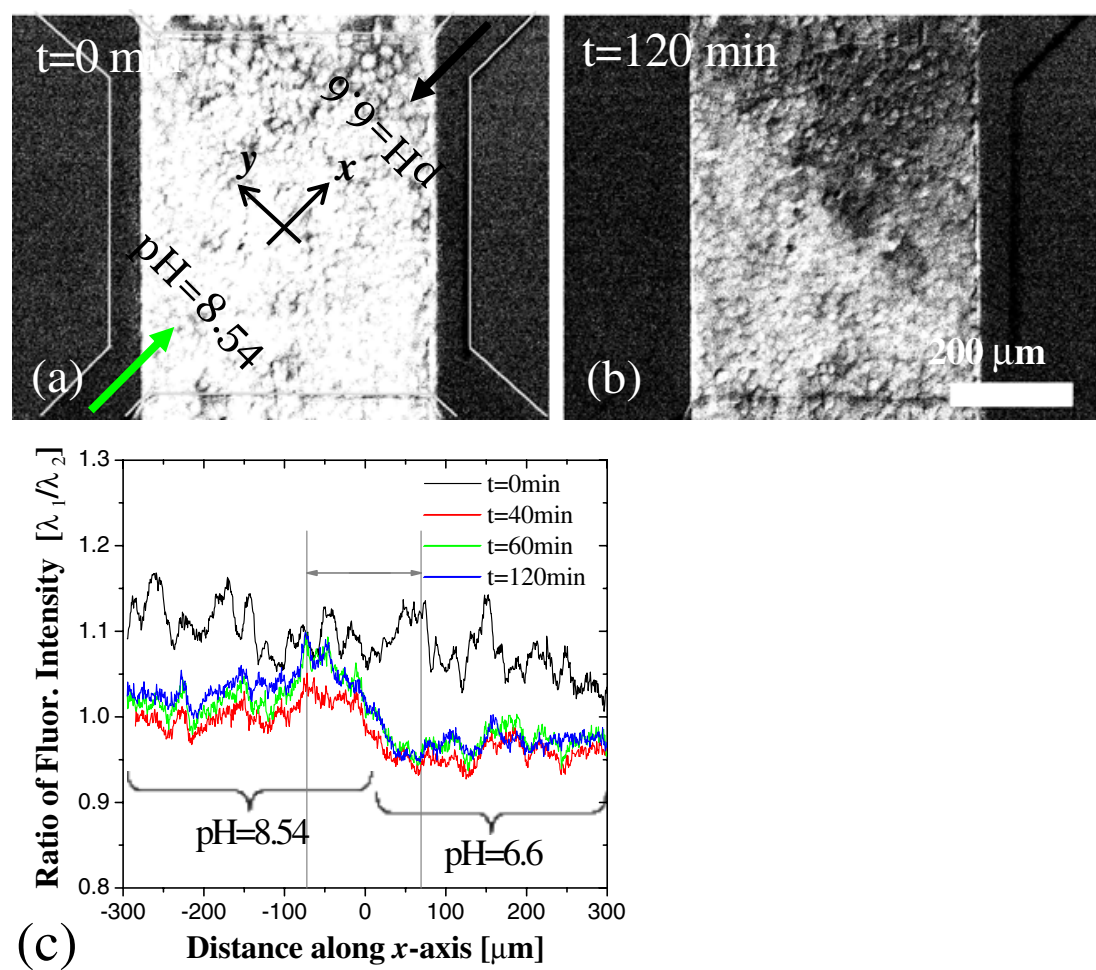

(c)

Distance along $x$-axis $[\mu \mathrm{m}]$

taken up. Last, the presence of adherent cells on the membrane was necessary to appropriately include their effect on mass transfer. Both white light nor fluorescent inverted microscopy of cells through the membrane was possible although we note that the top PDMS layers are transparent and upright microscopy would have been possible if needed.

\subsection{A $\sim 17$ Da molecule gradient can be mapped across} the membrane onto HeLa cells in culture

Given that BCECF fluorescence is $\mathrm{pH}$-sensitive, we modified the same assay to quantify how sharply gradients of extremely small molecules could be imposed on the cell culture. As in the previous experiment, we loaded cells into the top channel and then seeded them on the membrane. We then flowed DMEM/BCECF AM through the top channel, uniformly loading the cells in the entire chamber with the BCECF indicator [Fig. 6(a)]. After rinsing and filling the top channel with $\sim 1 \mathrm{ml}$ of a PBS buffer solution $(\mathrm{pH}=7.6)$ to remove any BCECF AM residue, we flowed DI water $\left(Q_{2}=1 \mathrm{ml} / \mathrm{h}\right.$, $\mathrm{pH}=6.6)$ and an HBSS solution $\left(Q_{3}=1 \mathrm{ml} / \mathrm{h}, \mathrm{pH}=8.54\right)$ through the bottom channel to generate a $\mathrm{pH}$ gradient. After $60 \mathrm{~min}$, two different zones of pHi can be seen in BCECFloaded cells; the profile reached steady-state at $t=120 \mathrm{~min}$. The quantified results of Fig. 6(c) are in good agreement with Fig. 6(b) and the distinct interface thickness was measured to be $\sim 140 \mu \mathrm{m}$. As expected, the interface is

Fig. 7 By controlling the flow rates of $Q_{2}$ and $Q_{3}$ and hydrodynamic resistances of $Q_{1}$ and $Q_{3}$ channels individually, asymmetric regions can be generated in (a) and (b)
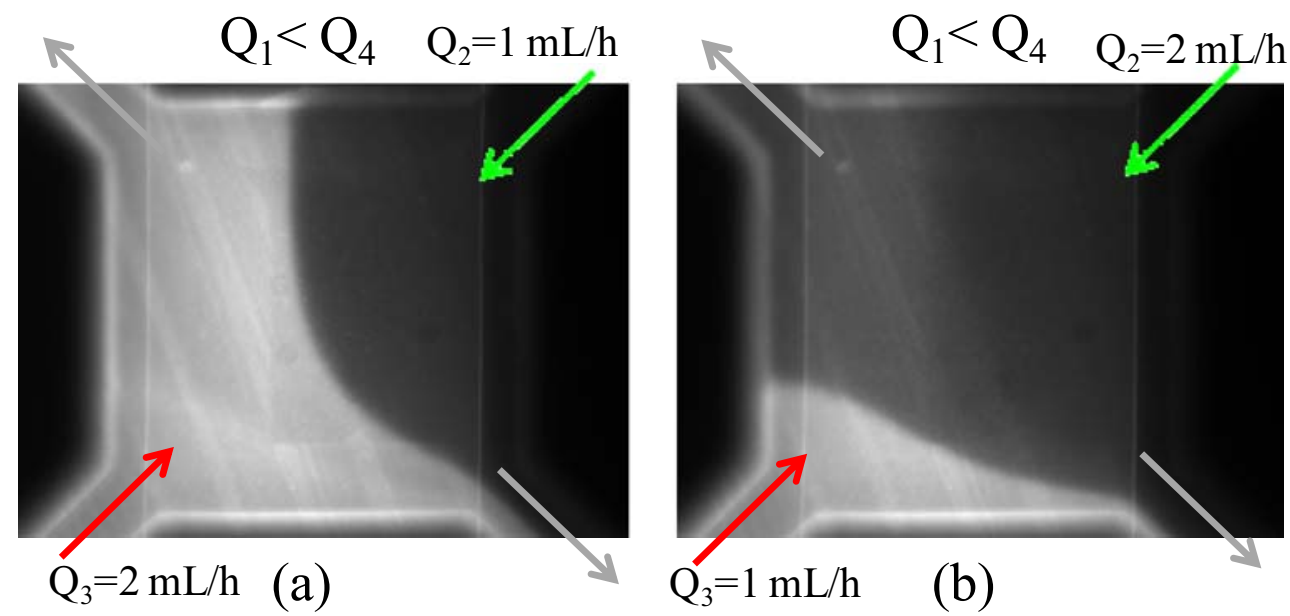
much wider than BCECF gradient results due to the higher diffusion constant of the hydroxyl ion compared to BCECF.

3.6 Asymmetric gradients and time-varying interfaces can be set by varying flow ratios

Lastly, we demonstrated that asymmetric gradients and timedependent chemical patterns could be generated by individually manipulating the flow rates and/or hydraulic resistances of each channel (Fig. 7). Flow rates are easily controlled using a syringe pump and hydraulic resistances are adjusted by varying device channel lengths. Hydraulic resistance $(R)$ is linearly proportional to the length of a channel $\left(R=\eta L / D_{\mathrm{h}}^{2} / A_{\mathrm{x}}\right)$, where $\eta$ is viscosity of a solution, $L$ is the length, $D_{\mathrm{h}}$ is a hydraulic diameter, and $A_{\mathrm{x}}$ is the crosssectional area of a channel); effective channel length could be varied by placing the tubing outlet holes at different positions along the outlet microchannel (Kim et al. 2007). In this way, we demonstrated that asymmetric patterns can be achieved by controlling the flow rates as well as by drilling output holes of $\mathrm{Q}_{1}$ and $\mathrm{Q}_{4}$ at different positions to change $L$ [Fig. 7(a) and (b)].

\section{Conclusions}

We demonstrated that our microfluidic device makes it possible to generate steep gradients of small molecules such as rhodamine-B (400 Da), $\mathrm{Ca}^{2+}$ ions (40 Da), BCECF AM (689 Da) and even hydroxyl ions ( $17 \mathrm{Da}$ ) over large spans of cell culture by confronting flows. This method facilitates the generation of uniform, stable gradients along the boundary of two fluids, offering a wide assay area for investigating effects of signaling molecules on cells growth. The use of a membrane to separate the gradient generation area from the cell culture area effectively eliminates or reduces shear forces on the cells. We successfully demonstrated that small molecule gradients could be mapped onto HeLa cells using both BCECF and different $\mathrm{pH}$ buffers. Lastly, we demonstrated how differential flow rates and fluidic resistances can change the position and shape of the interface region. We believe our approach could be useful in the study of small molecule gradients in developmental biology.

Acknowledgements We thank Keck Foundation for financial support. We also thank Jaehyun Park, Whijae Roh and Prof. Shuichi Takayama for helpful discussions on various aspects of cell culture, FEM simulation, and device design.

\section{References}

V.V. Abhyankar, M.A. Lokuta, A. Huttenlocher, D.J. Beebe, Characterization of a membrane-based gradient generator for use in cell-signaling studies Lab. Chip. 6(3), 389-393 (2006) doi:10.1039/b514133h
H.L. Ashe, J. Briscoe, The interpretation of morphogen gradients Development 133(3), 385-394 (2006) doi:10.1242/dev.02238

T. Bansal, M.P. Chang, M.M. Maharbiz, A class of low voltage, elastomer-metal 'wet' actuators for use in highdensity microfluidics Lab. Chip 7(2), 164-166 (2007) doi:10.1039/b614419e

S. Boyden, Chemotactic Effect of Mixtures of Antibody and Antigen on Polymorphonuclear Leucocytes J. Exp. Med 115(3), 453 (1962) doi:10.1084/jem.115.3.453

M.R. Bringer, C.J. Gerdts, H. Song, J.D. Tice, and R.F. Ismagilov, Microfluidic systems for chemical kinetics that rely on chaotic mixing in droplets. Philo Trans R Soc Lond A Math Phys Eng Sci 362(1818), 1087-1104 (2004).

K. Campbell, A. Groisman, Generation of complex concentration profiles in microchannels in a logarithmically small number of steps Lab. Chip 7(2), 264-272 (2007) doi:10.1039/b610011b

S. Chellam, M.R. Wiesner, C. Dawson, Slip at a uniformly porous boundary-effect on fluid-flow and mass-transfer J. Eng. Math 26(4), 481-492 (1992)

B.H. Chueh, D.G. Huh, C.R. Kyrtsos, T. Houssin, N. Futai, S. Takayama, Leakage-free bonding of porous membranes into layered microfluidic array systems Anal. Chem 79(9), 35043508 (2007) doi:10.1021/ac062118p

B.G. Chung, L.A. Flanagan, S.W. Rhee, P.H. Schwartz, A.P. Lee, E.S. Monuki et al., Human neural stem cell growth and differentiation in a gradient-generating microfluidic device Lab Chip 5(4), 401406 (2005) doi:10.1039/b417651k

B.G. Chung, F. Lin, N.L. Jeon, A microfluidic multi-injector for gradient generation. Lab. Chip 6(6), 764-768 (2006) doi:10.1039/b512667c

E.L. Cussler, Diffusion: mass transfer in fluid systems (Cambridge University Press, New York, 1997)

S.K.W. Dertinger, D.T. Chiu, N.L. Jeon, G.M. Whitesides, Generation of gradients having complex shapes using microfluidic networks Anal. Chem 73(6), 1240-1246 (2001) doi:10.1021/ac001132d

D.C. Duffy, J.C. McDonald, O.J.A. Schueller, G.M. Whitesides, Rapid prototyping of microfluidic systems in poly(dimethylsiloxane) Anal. Chem 70(23), 4974-4984 (1998) doi:10.1021/ac980656z

J. El-Ali, P.K. Sorger, K.F. Jensen, Cells on chips Nature 442(7101), 403-411 (2006) doi:10.1038/nature05063

M.E. Elsabban, B.U. Pauli, Cytoplasmic dye transfer between metastatic tumor-cells and vascular endothelium J. Cell Biol 115(5), 1375-1382 (1991) doi:10.1083/jcb.115.5.1375

J.B. Gurdon, P.Y. Bourillot, Morphogen gradient interpretation Nature 413(6858), 797-803 (2001) doi:10.1038/35101500

A.L. Horvath, Handbook of aqueous electrolyte solutions: physical properties, estimation and correlation methods (Wiley, New York, NY, 1985)

D. Irimia, D.A. Geba, M. Toner, Universal microfluidic gradient generator Anal. Chem 78(10), 3472-3477 (2006) doi:10.1021/ac0518710

R.F. Ismagilov, J.M.K. Ng, P.J.A. Kenis, G.M. Whitesides, Microfluidic arrays of fluid-fluid diffusional contacts as detection elements and combinatorial tools Anal. Chem 73(21), 5207-5213 (2001) doi:10.1021/ac010502a

N.L. Jeon, S.K.W. Dertinger, D.T. Chiu, I.S. Choi, A.D. Stroock, G. M. Whitesides, Generation of solution and surface gradients using microfluidic systems Langmuir 16(22), 8311-8316 (2000) doi:10.1021/la000600b

N.L. Jeon, H. Baskaran, S.K.W. Dertinger, G.M. Whitesides, L. Van de Water, M. Toner, Neutrophil chemotaxis in linear and complex gradients of interleukin- 8 formed in a microfabricated device Nat. Biotechnol 20(8), 826-830 (2002)

T.M. Keenan, A. Folch, Biomolecular gradients in cell culture systems Lab. Chip 8, 34-57 (2008) doi:10.1039/b711887b

T.M. Keenan, C.H. Hsu, A. Folch, Microfluidic "jets" for generating steady-state gradients of soluble molecules on open surfaces Appl. Phys. Lett 89(11) (2006) doi:10.1063/1.2345914 
A. Khademhosseini, R. Langer, J. Borenstein, J.P. Vacanti, Microscale technologies for tissue engineering and biology Proc. Natl. Acad. Sci. USA 103(8), 2480-2487 (2006) doi:10.1073/ pnas.0507681102

A. Kicheva, P. Pantazis, T. Bollenbach, Y. Kalaidzidis, T. Bittig, F. Julicher et al., Kinetics of morphogen gradient formation Science 315(5811), 521-525 (2007) doi:10.1126/science.1135774

T. Kim, E. Meyhofer, E.F. Hasselbrink, Biomolecular motor-driven microtubule translocation in the presence of shear flow: modeling microtubule deflection due to shear. Biomed. Microdevices 9(4), 501-511 (2007) doi:10.1007/s10544-007-9057-3

A.D. Lander, Morpheus unbound: Reimagining the morphogen gradient Cell 128(2), 245-256 (2007) doi:10.1016/j. cell.2007.01.004

A.D. Lander, Q. Nie, F.Y.M. Wan, Do morphogen gradients arise by diffusion? Dev. Cell 2(6), 785-796 (2002) doi:10.1016/S15345807(02)00179-X

F.Q. Nie, M. Yamada, J. Kobayashi, M. Yamato, A. Kikuchi, T. Okano, On-chip cell migration assay using microfluidic channels Biomaterials 28(27), 4017-4022 (2007) doi:10.1016/j.biomaterials.2007.05.037

L.A. Raftery, D.J. Sutherland, Gradients and thresholds: BMP response gradients unveiled in Drosophila embryos Trends Genet 19(12), 701-708 (2003) doi:10.1016/j.tig.2003.10.009

S.A. Rani, B. Pitts, P.S. Stewart, Rapid diffusion of fluorescent tracers into Staphylococcus epidermidis biofilms visualized by time lapse microscopy Antimicrob. Agents Chemother 49(2), 728-732 (2005) doi:10.1128/AAC.49.2.728-732.2005

W. Saadi, S.J. Wang, F. Lin, N.L. Jeon, A parallel-gradient microfluidic chamber for quantitative analysis of breast cancer cell chemotaxis Biomed. Microdevices 8(2), 109-118 (2006) doi:10.1007/s10544-006-7706-6
O. Shimmi, M.B. O'Connor, Physical properties of Tld, Sog, Tsg and Dpp protein interactions are predicted to help create a sharp boundary in Bmp signals during dorsoventral patterning of the Drosophila embryo Development 130(19), 4673-4682 (2003) doi:10.1242/dev.00684

S. Takayama, E. Ostuni, P. LeDuc, K. Naruse, D.E. Ingber, G.M. Whitesides, Laminar flows - subcellular positioning of small molecules Nature 411(6841), 1016-1016 (2001) doi:10.1038/35082637

G.M. Walker, J.Q. Sai, A. Richmond, M. Stremler, C.Y. Chung, J.P. Wikswo, Effects of flow and diffusion on chemotaxis studies in a microfabricated gradient generator Lab. Chip 5(6), 611-618 (2005) doi:10.1039/b417245k

Y. Wang, T. Mukherjee, Q. Lin, Systematic modeling of microfluidic concentration gradient generators J. Micromech. Microeng 16 (10), 2128-2137 (2006) doi:10.1088/0960-1317/16/10/029

I.D. Weiner, L.L. Hamm, Use of fluorescent dye BCECF to measure intracellular $\mathrm{pH}$ in cortical collecting tubule Am. J. Physiol 256 (5), F957-F964 (1989)

H.M. Yu, I. Meyvantsson, I.A. Shkel, D.J. Beebe, Diffusion dependent cell behavior in microenvironments Lab. Chip 5(10), 1089-1095 (2005) doi:10.1039/b504403k

X.Y. Zhu, L.Y. Chu, B.H. Chueh, M.W. Shen, B. Hazarika, N. Phadke et al., Arrays of horizontally-oriented mini-reservoirs generate steady microfluidic flows for continuous perfusion cell culture and gradient generation Analyst (Lond.) 129(11), 1026-1031 (2004) doi:10.1039/b407623k

D. Zicha, G.A. Dunn, A.F. Brown, A new direct-viewing chemotaxis chamber J. Cell Sci 99, 769-775 (1991)

S.H. Zigmond, Ability of polymorphonuclear leukocytes to orient in gradients of chemotactic factors J. Cell Biol 75(2), 606-616 (1977) doi:10.1083/jcb.75.2.606 